Check for updates

Cite this: RSC Adv., 2017, 7, 26680

Received 8th March 2017 Accepted 11th May 2017

DOI: $10.1039 / \mathrm{c} 7 \mathrm{ra0} 2828 \mathrm{~h}$

rsc.li/rsc-advances

\section{Aloe vera assisted facile green synthesis of reduced graphene oxide for electrochemical and dye removal applications $\uparrow$}

\author{
Gourav Bhattacharya, ${ }^{a}$ Shrawni Sas, ${ }^{b}$ Shikha Wadhwa, ${ }^{\text {b }}$ Ashish Mathur, ${ }^{\text {b }}$ \\ James McLaughlin ${ }^{c}$ and Susanta Sinha Roy (iD *a
}

\begin{abstract}
In the present work, the suitability of Aloe vera (AV) as a 'green reducing agent' has been investigated for the reduction of graphene oxide (GO). The extent of reduction was studied by varying the amount of AV. The physical and chemical properties of the GO and reduced graphene oxide (rGO) were investigated using UVVis spectrophotometry, FT-IR spectroscopy, High Resolution Transmission Electron Microscopy (HRTEM) and Selected Area Electron Diffraction (SAED). Partially reduced graphene oxide sheets obtained with $7.5 \mathrm{~g}$ of $\mathrm{AV}$ ( $\mathrm{rGO}-7.5$ ) demonstrated a maximum reduction efficiency of about $73 \%$ as evident from $\mathrm{FT}$-IR data. Cyclic voltammetry and electrochemical impedance spectroscopy studies revealed a significant enhancement in current density and a decrease in charge transfer resistance for the rGO-7.5 sample. Moreover, the as prepared rGO-7.5 sample showed a remarkable dye removal ability with a maximum efficiency of $\sim 98 \%$. The enhanced surface area, $\pi-\pi$ interaction and strong electrostatic attraction were correlated with the dye removal capability. The adsorption kinetics were also studied and pseudo second order adsorption phenomena were confirmed. The recyclability of the rGO-7.5 sample was further investigated and an excellent desorption capability was established.
\end{abstract}

\section{Introduction}

The striking thermal, electrical and mechanical properties of graphene have attracted immense attention. ${ }^{1-3}$ Among several synthesis techniques, chemical reduction of graphene oxide is one of the most popular methods. Chemical reduction is in general carried out using reducing agents such as hydrazine or sodium borohydride. ${ }^{4-6}$ Despite being a simple process, this method has some inherent disadvantages. High costs and the highly toxic nature of reducing agents, ${ }^{7}$ and the irreversible aggregation of graphene oxide (GO) restrict the usage of this method. To overcome these challenges, various green synthesis routes and bio-reduction methods are being explored, where plants extracts or microorganisms act as eco-friendly reducing agents. ${ }^{8-10}$ The use of micro-organisms requires aseptic conditions for their growth, ${ }^{\mathbf{1 1}}$ thus making the maintenance work more cumbersome. The use of plant extracts for reduction of GO eliminates the need of toxic reducing agents, making the

${ }^{a}$ Department of Physics, School of Natural Sciences, Shiv Nadar University, Gautam Buddha Nagar, 201314, Uttar Pradesh, India.E-mail: susanta.roy@snu.edu.in

${ }^{b}$ Amity Institute of Nanotechnology, Amity University Uttar Pradesh, Noida-201313, India

${ }^{c}$ Nanotechnology and Integrated Bioengineering Centre, Jordanstown Campus, University of Ulster, Newtownabbey, BT37 OQB, Northern Ireland, UK

$\dagger$ Electronic supplementary information (ESI) available. See DOI: $10.1039 / \mathrm{c} 7 \mathrm{ra02828h}$ process eco-friendly and cost-effective. They also help in controlling the crystal growth due to a slower reaction rate. ${ }^{\mathbf{1 2}}$ Various plant sources such as Citrus sinensis (orange peel), ${ }^{13}$ leaf extracts of Colocasia esculenta ${ }^{\mathbf{1 4}}$ and Mesua ferrea Linn, ${ }^{\mathbf{1 4}}$ grape extract $^{15}$ are pertinent to mention. Al-Marii et al. ${ }^{16}$ prepared highly reduced graphene (HRG)-Ag nanocomposite (NP) using Pulicaria glutinosa plant, which acted as a reducing agent and a ligand, which helped in the binding of silver NPs onto HRG layers. Local availability, cost-effectiveness and eco-friendly nature of plant extracts make them a decent alternative to harmful chemicals for the reduction of graphene oxide. Though there are several different plants or fruit extracts that have been used in the reduction process but global unavailability and not so efficient reduction capability restrict their usage.

The reduced graphene oxide (rGO) obtained by green synthesis route, has been utilized as a multifunctional material and are used in electrochemical charge storage, sensing, photocatalysis, dye removal and several other applications. ${ }^{17-20}$ Bo et al. ${ }^{21}$ synthesized rGO utilizing caffeic acid as a reducing agent and used the rGO for sensing and electrochemical storage purpose. Zhang et al. ${ }^{22}$ utilized L-cysteine, an amino acid to synthesize rGO and explored its photocatalytic activity on methylene blue (MB). Several industrial dye molecules are contaminating water and creating serious environmental pollution. ${ }^{23}$ Removal of these molecules from water is a big challenge. Dye adsorption ${ }^{24}$ is one of the most popular technique to recycle contaminated water. Recently, rGO has become 
a promising dye adsorbent material. ${ }^{25,26}$ Suresh et al. utilized clove $^{27}$ and cinnamon ${ }^{28}$ extracts to reduce graphene and studied its dye adsorption capability.

Aloe vera (AV), which belongs to the family Liliaceae, is one of the most widely used medicinal plant across the globe. It naturally grows across continents. In addition, it is one of the most cultivated plant and even found in the household due to its medicinal values. It is also used as an ornamental plant. It consists of 6 anti-septic agents, 23 polypeptides, at least 3 antiinflammatory agents and various vitamins such as vitamin A and vitamin B12 (ref. 29). AV leaf gel contain phytochemicals such as acetylated mannans, polymannans, anthraquinone Cglycosides, anthrones, other anthraquinones, including emodin, and various lectins. ${ }^{30} \mathrm{AV}$ extract has demonstrated an excellent capability as a reducing agent in the synthesis of triangular and spherical shaped gold and silver nanoparticles from their respective salts. ${ }^{31}$ Other nanoparticles such as indium oxide $\left(\operatorname{In}_{2} \mathrm{O}_{3}\right)^{32}$ has also been produced. Such reports emphasize the immense potential of AV as a reducing agent. Since AV is easily available, and has low cost, ${ }^{33}$ it is used as a substitute for hazardous chemicals for the reduction of metal salts.

In this work, the reducing capability of $\mathrm{AV}$ for the green reduction of graphene oxide has been investigated. AV extract contains manifold of organic compounds e.g. anthaquinones, polysaccharides, sugars etc. which have the natural reducing ability. Proposed synthesis mechanism to reduce GO through $\mathrm{AV}$ is environmental friendly and promising for the large-scale cost effective production of rGO. AV amount is one of the key parameter which commands the reduction profile and thus a systematic parametric investigation was performed to check the extent of reduction with different AV concentration. Fourier transformed infrared (FT-IR) spectroscopy has been utilized to find out the reduction efficiency. Cyclic voltammetry (CV) and electrochemical impedance spectroscopy (EIS) techniques have been employed to study electrochemical properties of the GO and rGO. The capability of as prepared rGO in dye adsorption was also investigated. De-coloration and dye removal study of methylene blue (MB), which is extensively used in dye industry to dye cotton, woods etc. was performed using rGO.

\section{Materials and methods}

\subsection{Materials}

Graphite powder and sodium nitrate were brought from $\mathrm{CDH}$. Ethanol (99\%, Sigma-Aldrich), sulphuric acid (98\%), potassium permanganate $(99.5 \%)$ and hydrogen peroxide $(30 \%)$ were brought from Merck, ethanol (99\%) and methanol were brought from Sigma-Aldrich. All the chemicals were of AR grade and were used without any further purification. Aloe vera gel in pure form was procured from local organic farming. All the solutions were made using de-ionised (DI) water $(18 \mathrm{M} \Omega \mathrm{cm})$.

\subsection{Synthesis}

2.2.1. Synthesis of graphene oxide (GO). GO was synthesized using modified Hummer's method. ${ }^{34-36}$ In a typical method, $1 \mathrm{~g}$ of graphite powder was mixed with $0.5 \mathrm{~g}$ of sodium nitrate $\left(\mathrm{NaNO}_{3}\right) .25 \mathrm{~mL}$ of sulphuric acid $\left(\mathrm{H}_{2} \mathrm{SO}_{4}\right)$ was added to the mixture followed by slow addition of $3 \mathrm{~g}$ of potassium permanganate $\left(\mathrm{KMnO}_{4}\right)$ over a period of 2 hours in an ice bath. The solution was stirred for 2 hours on ice bath. $500 \mathrm{~mL}$ of DI water was poured into the solution followed by very slow addition of $10 \mathrm{~mL}$ of $30 \%$ hydrogen peroxide $\left(\mathrm{H}_{2} \mathrm{O}_{2}\right)$ with vigorous stirring over ice bath. The as obtained yellow colored GO was then centrifuged and washed repeatedly with DI water and 50\% methanol until neutral $\mathrm{pH}$ was obtained. Finally, GO powder was dried in air.

2.2.2. Reduction of graphene oxide. To $80 \mathrm{~mL}$ distilled water, $0.04 \mathrm{~g}$ of GO powder was added and the suspension was ultra-sonicated for about $30 \mathrm{~min}$. As purchased AV with different masses (3, 7.5 and $12 \mathrm{~g}$ ) were added to $10 \mathrm{~mL}$ deionized water and stirred for $10 \mathrm{~min}$. AV solution was then added to the GO suspension and was refluxed for $24 \mathrm{~h}$ at $95{ }^{\circ} \mathrm{C}$. After the reaction, the suspension was centrifuged at ca. $3000 \mathrm{rpm}$. The black powder thus obtained was washed with distilled water many times, then with ethanol and dried in air. In order to obtain the maximum efficient reduction, the amount of AV was varied.

\subsection{Experimental}

2.3.1. Morphology analysis. Optical absorbance in the wavelength range $200-800 \mathrm{~nm}$ was measured using UV-Vis Spectrophotometer (UV-2600 Spectrophotometer, Shimadzu). FT-IR spectra was measured using Thermo Fisher Scientific instrument in the range $\sim 4000$ to $650 \mathrm{~cm}^{-1}$ at ambient condition, with a resolution of $\pm 4 \mathrm{~cm}^{-1}$ utilizing the transmission mode. The spectra was analyzed using Thermo Insight software. HRTEM images were taken using Fei-Philips, Morgagni 268D TEM (Digital TEM with image analysis system and maximum magnification $=\times 280000$ ) operating at a voltage $100 \mathrm{kV}$.

2.3.2. Electrochemical measurement. Electrochemical measurements (CV and EIS) were performed on an Autolab Potentiostat Galvanostat PGSTAT 302N (Metrhom, Netherlands). For CV measurement, a three-electrode set up consisting of $\mathrm{Ag} / \mathrm{AgCl}$ as the reference electrode, platinum wire as the counter electrode and glassy carbon electrode (GCE) with dropdried GO and/or rGO as the working electrode, was utilized. The CV scans were carried out between $-0.6 \mathrm{~V}$ to $1.0 \mathrm{~V}$ using $0.1 \mathrm{M}$ aqueous $\mathrm{KCl}$ solution containing $5 \mathrm{mM} \mathrm{Fe}(\mathrm{CN})_{6}{ }^{3-/ 4-}$ redox couple. The impedance measurements were carried out in the FRA potential scan mode with a three-electrode set up. A $3 \mathrm{~mm}$ diameter GCE coated with GO and/or rGO as active material was used as the working electrode along with platinum wire as the counter electrode. The EIS measurements were carried out at room temperature in $0.1 \mathrm{M}$ aqueous $\mathrm{KCl}$ solution containing $5 \mathrm{mM} \mathrm{Fe}(\mathrm{CN})_{6}{ }^{3-/ 4-}$ redox couple. For measurements, a sinusoidal alternating current (a.c) perturbation voltage with root mean square (r.m.s) value of $10 \mathrm{mV}$ was applied while the frequency was varied from $1 \mathrm{~Hz}$ to $100 \mathrm{kHz}$. The spectrum was fitted using NOVA software.

2.3.3. Adsorptive removal of methylene blue. To study the dye removal capability of rGO, methylene blue dye was used. $20 \mathrm{mg}$ rGO-7.5 was added in $125 \mathrm{~mL}$ methylene blue (MB) 
solution (4 ppm). The mixture was shaken on an orbital shaker at $25{ }^{\circ} \mathrm{C}$ and at $150 \mathrm{rpm}$. A fixed volume of the dye solution was taken out after regular time intervals and centrifuged for the removal of rGO. Absorbance for all extracted dye samples was recorded at $664 \mathrm{~nm}^{37}$

Removal percentage (1) was estimated by using the following formula: ${ }^{38}$

$$
\text { Removal percentage }=\left\{\left(C_{0}-C_{t}\right) / C_{0}\right\} \times 100
$$

where $C_{0}$ is the initial concentration of dye and $C_{t}$ is concentration after a time interval $t$. The adsorption amount (2) $q_{t}$ was calculated using following equation: ${ }^{37}$

$$
q_{t}=\left[\left(C_{0}-C_{t}\right) V\right] / m
$$

where $q_{t}$ is the amount of adsorbed dye per gram of the adsorbent $\left(\mathrm{mg} \mathrm{g}^{-1}\right), C_{0}$ and $C_{t}$ are the concentrations of the $\mathrm{MB}$ solution $\left(\mathrm{mg} \mathrm{L}^{-1}\right)$ at the initial and adsorption time $t$, respectively. $V$ is the volume of the MB solution (L) and $m$ is the mass of the adsorbent $(\mathrm{g})$.

Dye removal capability of GO was also studied, keeping all the other parameters fixed.

2.3.4. Recycling study. The adsorption stability of rGO-7.5 was examined by recycling it for 5 separate MB adsorption studies as mentioned by Tiwari et al. ${ }^{26}$. In the actual experiment, $20 \mathrm{mg}$ rGO-7.5 was added in $125 \mathrm{~mL}$ of MB solution (4 ppm). The mixture was shaken at $25{ }^{\circ} \mathrm{C}$ and $150 \mathrm{rpm}$ for 15 minutes. After centrifugation, the rGO was removed from the solution.
Absorbance for the solution was recorded at $664 \mathrm{~nm}$. The extracted rGO was filtered and consequently washed with ethylene glycol (EG). ${ }^{26}$ The as washed rGO-7.5 was then dried and utilized for the second adsorption experiment. This process was repeated for 5 times.

\section{Results and discussions}

\subsection{Morphology analysis}

Fig. 1 shows the FT-IR spectra of GO and rGO samples. The presence of various oxygen functional groups in Fig. 1a confirm the successful oxidation of graphite to GO. The characteristic peaks of GO are observed at $1640 \mathrm{~cm}^{-1}(\mathrm{C}=\mathrm{O}$ stretching $)$ and at $1060 \mathrm{~cm}^{-1}$ (C-O stretching vibration mode), with a broad peak at $3250 \mathrm{~cm}^{-1}$ (O-H stretching vibrations). ${ }^{39}$ The disappearance of peak at $1060 \mathrm{~cm}^{-1}$ indicates a decrease of the $\mathrm{C}-\mathrm{O}$ stretching vibrations with increase in AV concentration. This observation supports the reduction of GO to rGO $^{40} \mathrm{~A}$ broad peak at 3250 $\mathrm{cm}^{-1}$ is flattened in the reduced samples due to removal of hydroxyl groups. ${ }^{38}$

From FT-IR peak area analysis at $1060 \mathrm{~cm}^{-1}$ (Fig. 1b and Table 1), the reduction in the peak area was observed with the increase of AV concentration. It can be seen that the curve was almost flattened for sample rGO-7.5. The peak at $1060 \mathrm{~cm}^{-1}$ as assigned to $\mathrm{C}-\mathrm{O}$ stretching vibration mode was therefore found to decrease, which suggests the reduction process. Area under peaks has been utilized to estimate the reduction efficiency by using following formula.
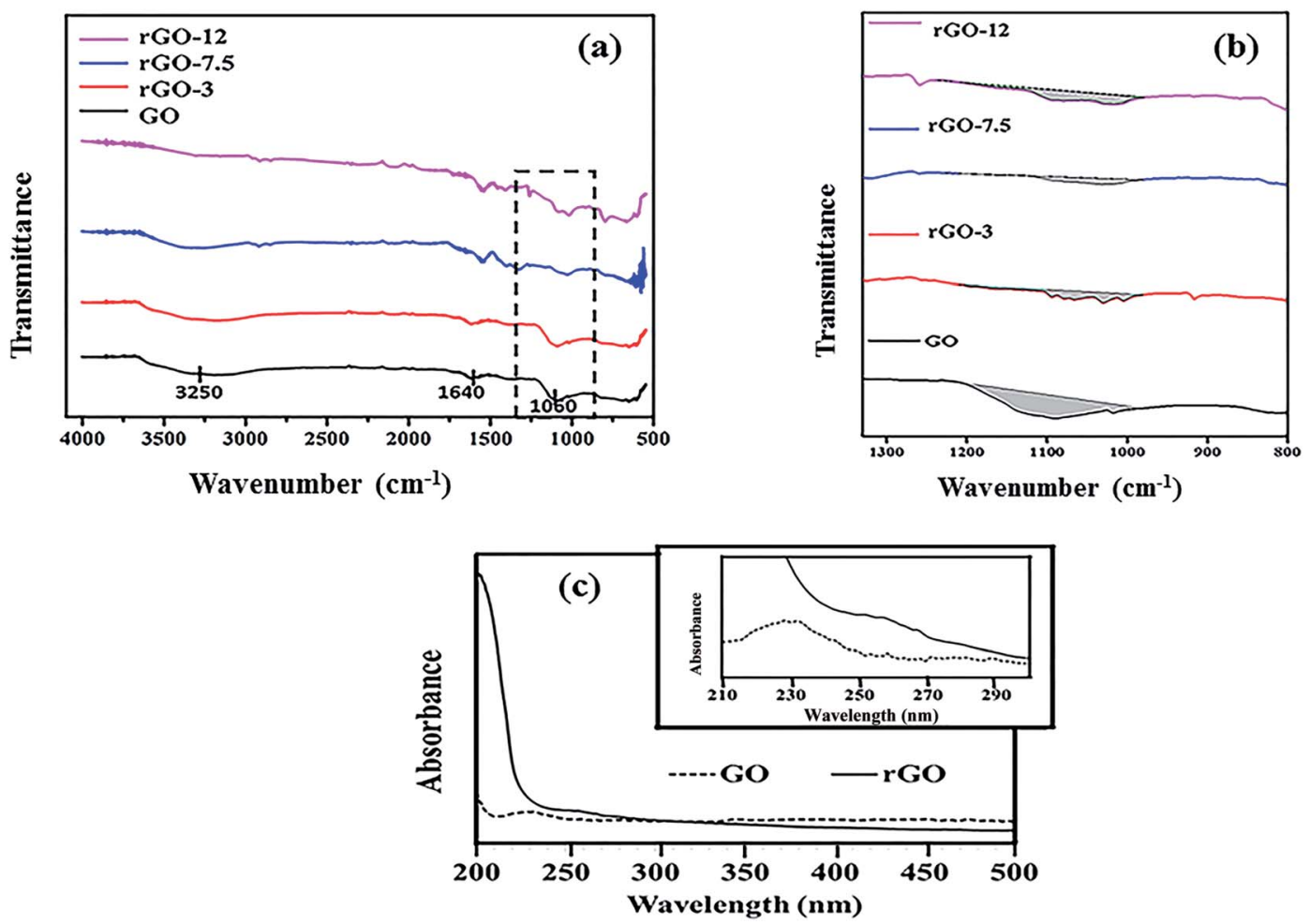

Fig. 1 (a) FT-IR spectra of GO, rGO-3, rGO-7.5 and rGO-12 samples. (b) Peak area analysis of FT-IR spectra at $1060 \mathrm{~cm}^{-1}$ and (c) UV-Vis spectrum of GO and rGO-7.5. Inset shows the UV-Vis spectrum between 210 and $300 \mathrm{~nm}$. 
Table 1 Analysis of peak area at $1060 \mathrm{~cm}^{-1}$ in FT-IR

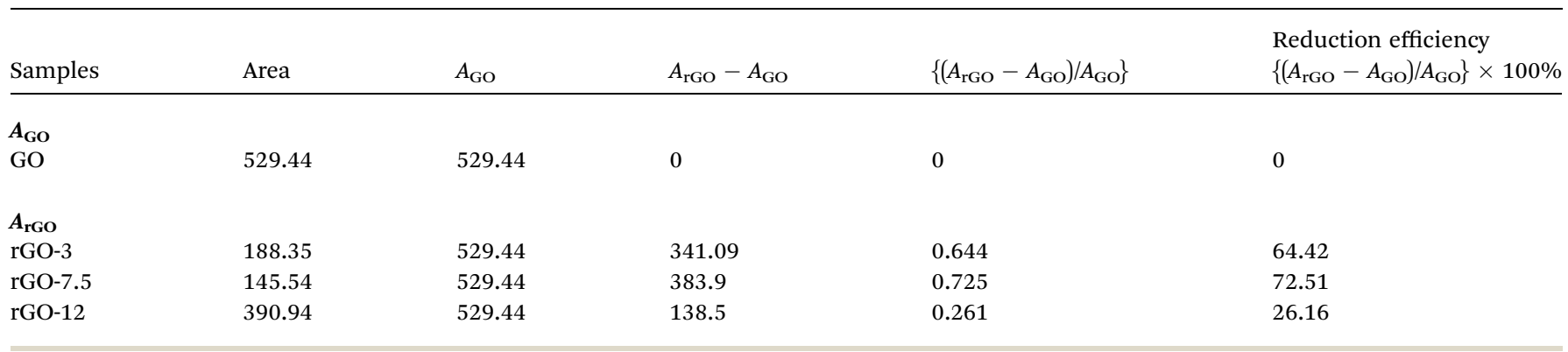

$$
\text { Reduction efficiency } \%=\left(\frac{A_{\mathrm{GO}}-A_{\mathrm{rGO}}}{A_{\mathrm{GO}}}\right) \times 100
$$

where, $A_{\mathrm{GO}}=$ area under peak for $\mathrm{GO}, A_{\mathrm{rGO}}=$ area under peak for rGO.

About $73 \%$ reduction of GO was achieved with $7.5 \mathrm{~g}$ of $\mathrm{AV}$ on the basis of FT-IR analysis. The absorption properties of nanoparticles can be understood well using UV-visible spectroscopy. In Fig. 1c, a characteristic peak of GO is visible at $230 \mathrm{~nm}$ which can be attributed to the $\pi-\pi^{*}$ transition of the aromatic $\mathrm{C}-\mathrm{C}$ ring, ${ }^{39}$ thus showing strong absorption. In case of rGO-7.5, the number of oxygen group decreases significantly as a result of which the electrons require very less energy for excitation. Due to this, absorption peak for rGO is shifted to $259 \mathrm{~nm}$.

Graphene oxide sheets were obtained after modified Hummer's synthesis from graphite powder. The material demonstrates presence of crystalline phases as indicated by bright spots from SAED (top right in Fig. S1 ESI $\dagger$ ). Reduced graphene oxide contains overlapped crumpled sheets and is represented in bottom left, Fig. S1 (ESI). $\uparrow$ The sheets appear folded from the edges. These folds and corrugations impart thermodynamic stability to the two-dimensional structures. ${ }^{\mathbf{4 1}} \mathrm{In}$ Fig. S1, $\uparrow$ less bright spots are observed from SAED of rGO-7.5 (bottom right). Due to the presence of crumpled and folded sheets, the electron diffraction from different crystalline planes were masked making the spots appear less bright.

\subsection{Electrochemical analysis}

3.2.1. Cyclic voltammetry (CV). The cyclic voltammetry of GO, rGO-3, rGO-7.5 and rGO-12 is represented in Fig. 2a. In case of GO modified GCE, very less current compared to the rGO specimens are observed. This slow electron transfer of redox couple $\mathrm{Fe}(\mathrm{CN})_{6}{ }^{3-/ 4-}$ in $\mathrm{GO}$ is associated with lower conductivity of GO and presence of large number of oxygen containing functional groups. On the other hand, CV scans of rGO showed a pair of prominent redox peaks suggesting increased electron transfer. However, among the rGO species, rGO-7.5 shows the highest current density. The electrochemical response of rGO7.5 modified GCE as a function of scan rate is further illustrated in Fig. 2b. An increase in the scan rate resulted a shift in the anodic peak potential towards positive side and cathodic peak potential towards negative potentials. Additionally, a linear increase in the redox peak current with the square root of scan rate for rGO-7.5 (Fig. 2c) is obtained. This linear behavior clearly suggests that the semi-infinite linear diffusion controls the redox reaction with a rapid electron transport ${ }^{42}$.

3.2.2. Impedance spectroscopy. In order to investigate the changes in the conductivity during the reduction of GO to rGO EIS measurements were carried out for both GO and rGO-7.5 sample. The Nyquist plot obtained through EIS measurement can give valuable information about the variation in the conductivity of electrode material. The diameter of the semicircle of Nyquist plot provides the magnitude of the charge transfer resistance. ${ }^{43}$ The Nyquist plots obtained for GO and rGO are shown in Fig. 2d, with the Nyquist plot for GO modified GCE electrode exhibiting a broad semicircle suggesting that the modification of electrode with GO obstruct the charge transfer due to poor conductivity of GO and repulsion between the surface charges of GO and electrolyte ions. However, for rGO modified electrode a sharp reduction in the semicircular diameter has been observed suggesting enhancement of charge transfer among electrolyte $\left(\left[\mathrm{Fe}(\mathrm{CN})_{6}\right]^{3-/ 4-}\right)$ and the electrode.

The EIS data was further fitted using a model equivalent electrical circuit (inset of Fig. 2d). The circuit contains a series resistance $\left(R_{\mathrm{S}}\right)$ which considers both solution resistance and contact resistance, charge transfer resistances $\left(R_{\mathrm{ct} 1}\right)$ and $\left(R_{\mathrm{ct} 2}\right)$ and constant phase elements $\left(Q_{1}, Q_{2}\right)^{\mathbf{4 4}}$ for working and counter electrodes respectively. This kind of electrical circuit is also reported in the literature. ${ }^{45}$ The fitted and the experimental data are plotted in Fig. $2 d$ and it shows residual $\chi^{2}$ values of $\sim 0.01$ (for GO) and $\sim 0.04$ (for rGO-7.5) confirming an excellent fitting. It is to be mentioned here that charge transfer resistance of the GO modified working electrode was $\sim 25 \mathrm{k} \Omega$ and the same was reduced to $\sim 9 \mathrm{k} \Omega$ for the rGO-7.5 modified electrode.

\subsection{Dye removal study}

3.3.1. Adsorption of $\mathrm{MB}$ by GO and rGO-7.5. In dye adsorption study, $125 \mathrm{~mL}$ of $\mathrm{MB}$ aqueous solution (4 ppm) was treated with $20 \mathrm{mg}$ GO and rGO-7.5 samples. Fig. 3a shows absorption spectra of dye solution treated with RGO-7.5 sample for different time durations. A sharp reduction in absorbance (around $664 \mathrm{~nm}$ ) indicates maximum removal of dye in first 5 minutes. Fig. $3 \mathrm{~b}$ depicts the percentage removal of $\mathrm{MB}$ with time. For both GO and rGO, most of the dye molecules were adsorbed while reaching equilibrium condition. $98 \%$ dye adsorption is achieved in case of rGO while, in case of GO it is found to be $\sim 80 \%$. 

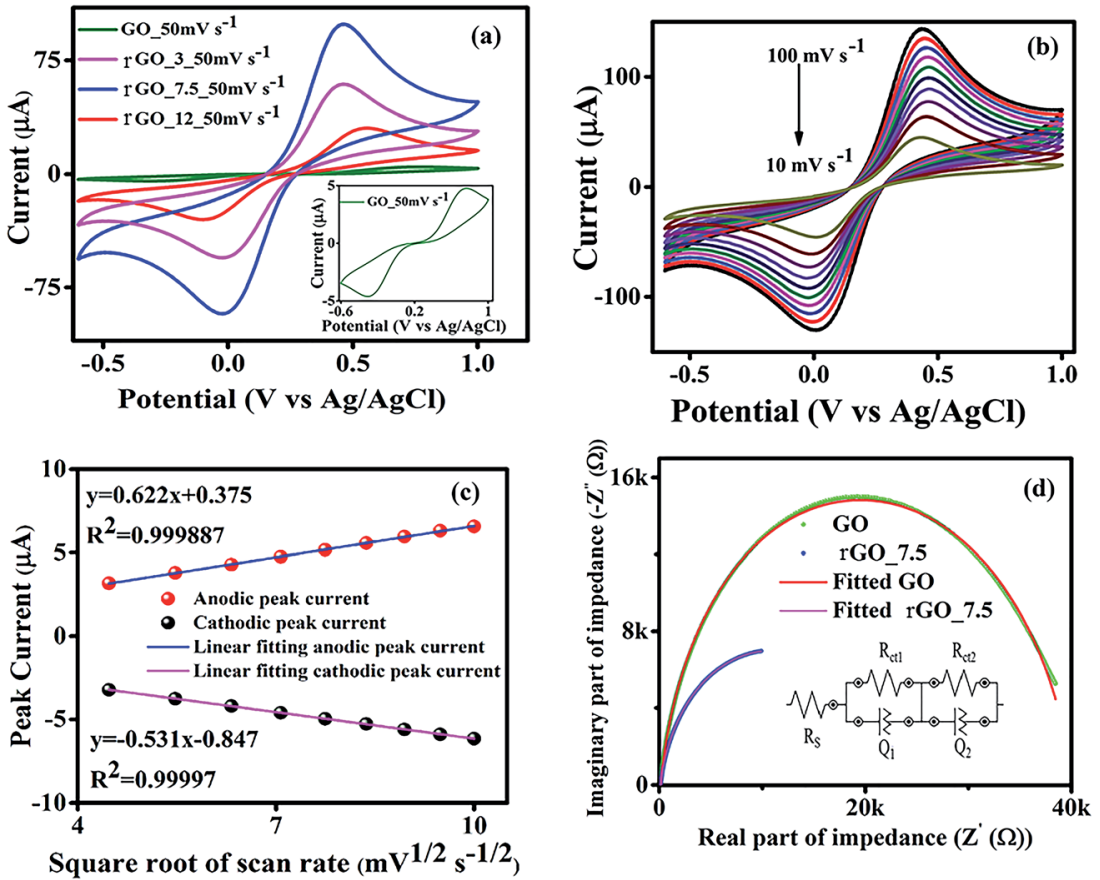

Fig. 2 (a) Cyclic voltammogram response of GO modified GCE, rGO-3 modified GCE, rGO-7.5 modified GCE, rGO-12 modified GCE in the solution of $0.1 \mathrm{M} \mathrm{KCl}$ containing $5 \mathrm{mM} \mathrm{Fe}(\mathrm{CN})_{6}{ }^{3-14-}$ with scan rate $50 \mathrm{mV} \mathrm{s}^{-1}$. Inset shows the cyclic voltammogram response of GO modified GCE, (b) cyclic voltammogram response of $\mathrm{rGO}-7.5$ modified GCE with scan rate $10-100 \mathrm{mV} \mathrm{s}{ }^{-1}$ in the solution of $0.1 \mathrm{M} \mathrm{KCl} \mathrm{containing} 5 \mathrm{mM}$ $\mathrm{Fe}(\mathrm{CN})_{6}{ }^{3-14-}$, (c) variation of anodic and cathodic peak currents as a function of scan rate for rGO-7.5 and (d) Nyquist plot of experimental and fitted impedance data for GO and rGO-7.5. (Inset of d) shows the equivalent electrical circuit.

Both GO and rGO have shown exceptional dye removal efficiency towards MB, a cationic organic dye. Strong $\pi-\pi$ interaction between $\mathrm{MB}$ and $\mathrm{rGO}(\mathrm{GO})$ surface is the prime reason of adsorption ${ }^{25}$ in addition to high electrostatic interaction. ${ }^{46}$ The effective surface area of rGO-7.5 sample is in general, much higher than that of GO and thus the contact area was enhanced significantly in case of rGO-7.5 which in turn boosted its adsorption efficiency. ${ }^{47}$ Also, it was reported earlier that the presence of trace amount of residual GO after centrifugation may reduce the adsorption efficiency ${ }^{15}$.

3.3.2. Kinetic study for the MB adsorption. The kinetics of adsorption of $\mathrm{MB}$ on $\mathrm{GO}$ and $\mathrm{rGO}$ in aqueous medium at a fixed initial concentration $(4 \mathrm{ppm})$ at room temperature $\left(25^{\circ} \mathrm{C}\right)$ were carried out to understand the adsorption process and represented in Fig. S2 of ESI. $\uparrow$ For both the samples, adsorption increases in the first 5 minutes, then slows down and reaches equilibrium after 10 minutes.

The kinetic parameters corresponding to adsorption of $\mathrm{MB}$ at the GO and rGO-water interface were assessed using pseudo first-order and pseudo second-order kinetic models with 4 ppm of initial dye concentration.

Lagergren pseudo first-order kinetic model (4) is expressed $\operatorname{as}^{48}$

$$
\log \left(q_{\mathrm{e} / \mathrm{expt}}-q_{t}\right)=\log q_{\mathrm{e} / \mathrm{fited}}-\left(k_{1} t / 2.303\right)
$$

where $k_{1}$ is the rate constant of the pseudo first-order kinetic equation and $q_{t}, q_{\mathrm{e} / \mathrm{expt}}$ and $q_{\mathrm{e} / \mathrm{fitted}}$ are the adsorption densities
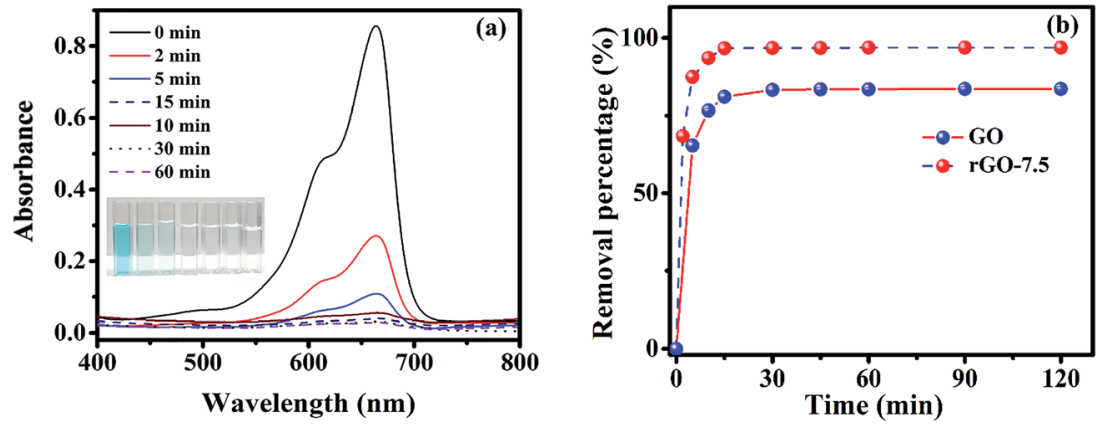

Fig. 3 Removal of methylene blue dye using rGO-7.5 samples. (a) Change in absorbance with time (inset shows the optical images of the solution after different time interval), (b) variation in removal percentage with time for GO, rGO-7.5. 
Table 2 Kinetic model parameters for the adsorption of MB onto the $\mathrm{GO}$ and $\mathrm{rGO}-7.5$

\begin{tabular}{lll}
\hline Kinetic model & GO & rGO-7.5 \\
\hline$q_{\mathrm{e} / \text { expt }}\left(\mathrm{mg} \mathrm{g}^{-1}\right)$ & 5.220 & 6.053 \\
& & \\
Pseudo first order & & \\
$k_{1}\left(\mathrm{~min}^{-1}\right)$ & 0.141 & 0.221 \\
$q_{\mathrm{e} / \mathrm{fitted}}\left(\mathrm{mg} \mathrm{g}^{-1}\right)$ & 2.236 & 2.275 \\
$R^{2}$ & 0.9730 & 0.9270 \\
& & \\
Pseudo second order & & 0.497 \\
$k_{2}\left(\mathrm{~g} \mathrm{mg}^{-1} \mathrm{~min}^{-1}\right)$ & 0.307 & 6.076 \\
$q_{\mathrm{e} / \mathrm{fitted}}\left(\mathrm{mg} \mathrm{g}^{-1}\right)$ & 5.255 & 0.9998 \\
$R^{2}$ & 0.9999 &
\end{tabular}

of $\mathrm{MB}$ at time $t$, at equilibrium (experimental and fitted) respectively.

$q_{\mathrm{e} / \text { fitterd }}$ was estimated from Fig. S3 of ESI $\dagger$ and is presented in Table 2 . The $R^{2}$ values for GO and rGO are $\sim 0.97$ and $\sim 0.93$ respectively. The estimated $q_{\mathrm{e} / \text { fitted }}$ values are much lower compared to the experimental values (Table 2).

The linear form of pseudo second-order kinetics is presented by $^{49}$ eqn (5) as

$$
t / q_{t}=\left(1 / k_{2} q_{\mathrm{e}}^{2}\right)+t / q_{\mathrm{e}}
$$

where $k_{2}$ is the pseudo second-order adsorption rate constant. Variation of $t / q_{t}$ with time for MB adsorption for GO and rGO-7.5 are shown in Fig. 4a and b respectively. The values of $k_{2}$ and $q_{\mathrm{e}}$ were evaluated from the slope and intercept of plots of $t / q_{t}$ versus $t$ (Fig. 4) and presented in Table 2. The $R^{2}$ values are much better compared to the pseudo first order kinetic model Table 2 . Also, the value of $q_{\mathrm{e}}$ nicely matches with the experimental data indicating that MB adsorption kinetics follows pseudo second order rate kinetics.

Intraparticle diffusion ${ }^{50}$ and Boyd kinetic ${ }^{51}$ models were further evaluated to understand the transport mechanism of adsorption process.

The intraparticle diffusion model is described by ${ }^{50}$ eqn (6) as

$$
q_{t}=k_{\mathrm{i}} t^{1 / 2}+c
$$

where $k_{\mathrm{i}}$ is the intraparticle diffusion rate constant and $c$ is a constant. The graph between $q_{t}$ and $t^{1 / 2}$ for rGO-7.5 samples were plotted in Fig. S4 of ESI. $\dagger$ The fitting was rather poor and also the fitted line in Fig. $\mathrm{S} 4 \uparrow$ do not pass through the origin suggestive of non-suitability of intraparticle diffusion as ratecontrolling step of adsorption process. To differentiate between external and intraparticle diffusion, the Boyd kinetic model was further utilized to determine the actual ratecontrolling step. The Boyd plot ( $B_{t} v s$. time) is made utilising the formula: ${ }^{51}$

$$
B_{t}=-0.4977-\ln (1-F)
$$

where

$$
F=q_{t} / q_{\mathrm{e}}
$$

and $B$ is the Boyd's number. The curves of the Boyd model plot (Fig. S5, ESI $\dagger$ ) do not pass through the origin, which indicates that external mass transport mainly governs the rate-limiting process of adsorption of MB onto GO and rGO. Similar results for adsorption of $\mathrm{MB}$ on activated carbon, was reported by $\mathrm{Ai}$ et $a .^{52}$

3.3.3. Recycling of rGO-7.5. Extreme environmental and economic demands of sustainability require recyclable adsorbent particles that can be utilized for several dye removal cycles. In addition to its high adsorption capability, a better desorption property of the particle is also preferred for practical applications. The regeneration and reuse of as prepared rGO sample was investigated and shown in Fig. 5. By utilizing a common, inexpensive solvent EG, rGO can be efficiently recycled. ${ }^{26}$ The percentage of capture is plotted against the cycle number. From the figure, it is quite clear that even after 5 adsorption cycles, $99 \%$ of initial dye removal capability can be attained. The dye removal efficiency decreased by $1 \%$ after 5 cycle of its usage (from $98 \%$ to $97 \%$ ). This shows the reusability and suitability of the adsorbent in practical industrial usage.

Reduced graphene oxide and its composites/derivatives have been explored by many researchers to study its dye removal capability. A comparative study of the dye adsorption of graphene based materials is represented in Table 3. From the table, it is observed that rGO and its composites have been used to remove many aqueous dye molecules. Many of the researchers used magnetic nanoparticles to separate out the composites and to reuse it. ${ }^{53,54}$ Our study shows that the as prepared rGO-7.5 exhibits very fast dye removal capability with $98 \%$ of the
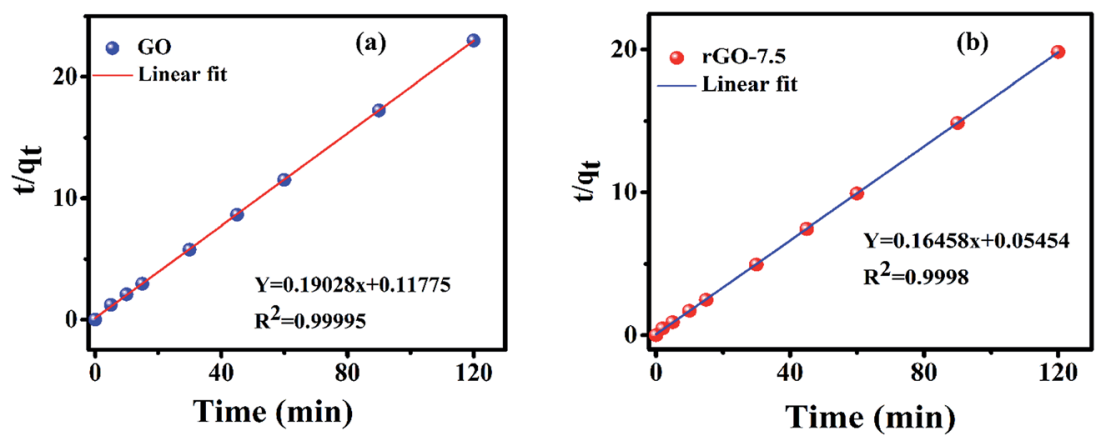

Fig. 4 Variation of $t / q_{t}$ vs. $t$ for MB adsorption. (a) For GO and (b) for rGO-7.5. 


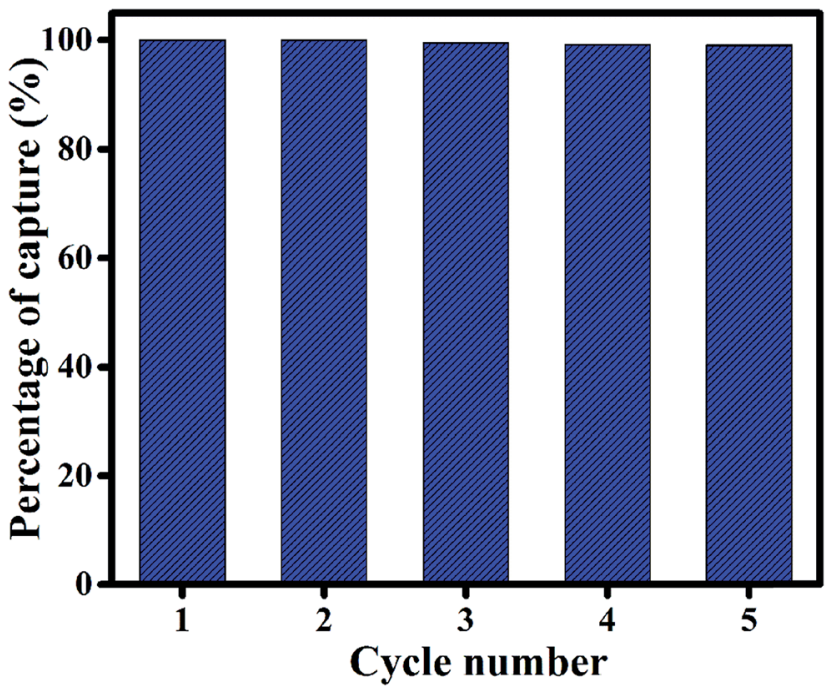

Fig. 5 Recycling performance of $\mathrm{rGO}-7.5$ for capturing methylene blue (MB) dye up to 5 cycles. The initial concentration of MB solution was taken as $4 \mathrm{ppm}$.

removal in 15 minutes. By utilizing an inexpensive solvent EG, we have successfully demonstrated the reusability of as prepared rGO-7.5 for up to 5 cycles without any significant change in adsorption.

\subsection{Mechanism of reduction of graphene oxide}

AV leaf extract contains mainly anthaquinones (phenolic compounds such as aloin and aloe-emodin), sugars (mainly monosaccharides (glucose and fructose) and polysaccharides (glucomannans/polymannose)), fatty acids, amino acids, minerals, vitamins and enzymes..$^{55}$ Composed mainly of polyphenols and sugars, AV thus demonstrates a mixture of chemical structures which can be easily oxidized. Among sugars, acemannan or acetylated mannan is the predominant
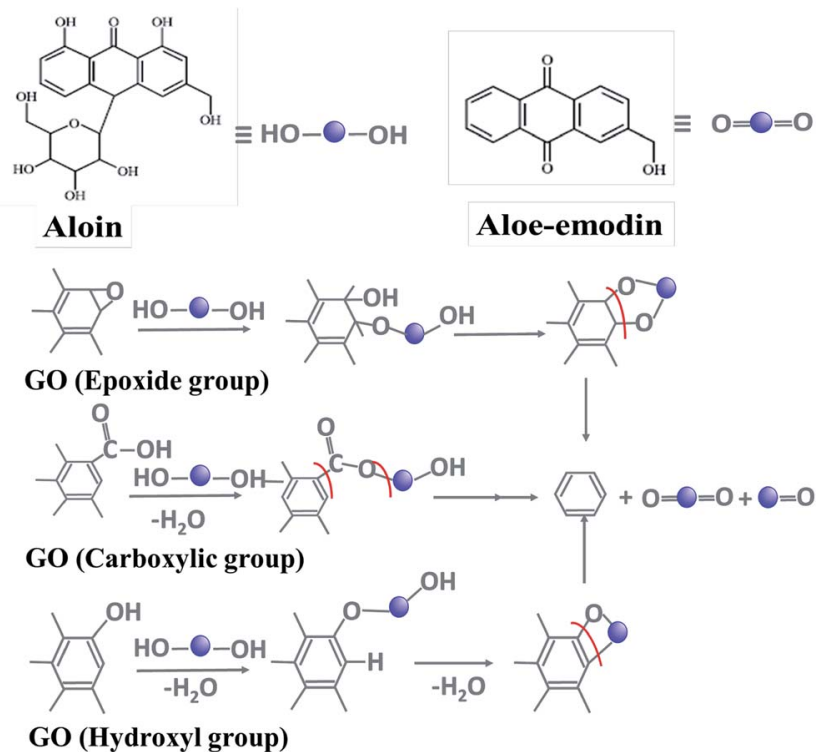

Fig. 6 Proposed reduction mechanism of GO.

component polysaccharide of AV gel. Other major components include anthraquinones and derivatives. ${ }^{42}$ The reduced forms contain polyhydroxy groups (e.g. acemannan) or polyphenols (e.g. anthraquinones) and the oxidized forms contain carbonyl groups (or anthraquinone backbone in aloe-emodin).

Graphene oxide contains a variety of functional moieties on its surface such as carboxylic, hydroxyl and epoxy groups. In the light of the detailed characterization of the rGO and GO samples, the mechanism of the reaction has been proposed. Carboxylic group undergoes condensation upon reaction with the reduced forms in AV to form an ester. Further reduction causes ring opening resulting in the formation of reduced form of graphene oxide. In case of hydroxyl derivative, condensation to form ring followed by ring cleavage facilitates the reduction of GO. Epoxy group undergoes ring opening upon reaction with

Table 3 Dye removal capability of reduced graphene oxide and its nanocomposites synthesized by using different reducing reagent

Material used

Magnetite/reduced graphene oxide Magnetite/reduced graphene oxide Graphene- $\mathrm{Fe}_{3} \mathrm{O}_{4} @$ ararbon (GFC) hybrids Reduced graphene oxide (RGO)-based hydrogels

Reduced graphene oxide (RGO)/ $\mathrm{Fe}_{3} \mathrm{O}_{4}$ nanocomposites

Chemically reduced graphene oxide

Copper-modified reduced graphene oxide Reduced graphene oxide

Graphene $/ \mathrm{Fe}_{3} \mathrm{O}_{4} /$ chitosan nanocomposite $\mathrm{SnO}_{2}$ quantum dots (QDs) decorated reduced graphene oxide Partially reduced graphene oxide

Reducing agent

Sodium acrylate and sodium acetate Sodium acrylate and sodium acetate Glucose

Sodium ascorbate

Solanum trilobatum extract

Concentrated ammonia solution (37 wt\%)

Sodium borohydride

Grapes (Vitis vinifera) extract

Ammonium acetate

A hybrid complex $\left[\left(\mathrm{N}_{2} \mathrm{H}_{4}\right)_{m}\left(\mathrm{SnCl}_{4}\right)_{n}\right]$

Aloe vera
Dye

Dye used

Rhodamine B

Malachite green Methylene blue Rhodamine B

Methylene blue

Methylene blue

Rhodamine B

Malachite green

Methylene blue

Methylene blue

Methylene blue concentration

\% removal

References
$5 \mathrm{ppm}$

$5 \mathrm{ppm}$

$50 \mathrm{ppm}$

$20.5 \mu \mathrm{M}$

$93.7 \mu \mathrm{M}$

$31.5 \mu \mathrm{M}$

$5.3 \mu \mathrm{M}$

$5 \mathrm{ppm}$

$25 \mathrm{ppm}$

$10 \mathrm{ppm}$

4 ppm

$\begin{array}{ll}91 & 53 \\ 94 & 53 \\ 97.8 & 54 \\ 97 & 26 \\ & \\ 95.18 & 18 \\ & \\ 84.8 & 56 \\ & \\ 86 & 57 \\ 90 & 15 \\ 98 & 58 \\ 94 & 59 \\ & \\ 98 & \text { Present } \\ & \text { work }\end{array}$


the polyhydroxy groups in AV followed by ring formation and cleavage to cause reduction of GO (Fig. 6).

\section{Conclusions}

Aloe vera is used as a green reducing agent for the reduction of graphene oxide. Optimum reduction efficiency of about $73 \%$ was achieved with $7.5 \mathrm{~g}$ of reducing agent. Interfacial charge transfer resistance substantially decreased in the rGO-7.5, due to the reduction of GO by Aloe vera. As obtained rGO also exhibited enhanced current density and higher conductivity than GO sample. Proposed reduction mechanism demonstrates the role of sugars and anthaquinones as major components in the reduction of graphene oxide. The rGO exhibited remarkable fast dye adsorption capability and the adsorption kinetics was found to be pseudo second order in nature, which was governed by external mass transport. Excellent dye adsorption-desorption capability and recyclability of synthesized rGO makes it a promising candidate for dye adsorbent material. This work illustrates a whole new 'green' approach of synthesizing large scale reduced graphene oxide, which may find suitability in electronics and sensing applications.

\section{Acknowledgements}

Gourav Bhattacharya is indebted to Shiv Nadar University for providing PhD scholarship.

\section{References}

1 A. A. Balandin, Nat. Mater., 2011, 10, 569-581.

2 K. S. Novoselov, V. Fal, L. Colombo, P. Gellert, M. Schwab and K. Kim, Nature, 2012, 490, 192-200.

3 X.-H. Lin and J.-G. Gai, RSC Adv., 2016, 6, 17818-17844.

4 S. Stankovich, D. A. Dikin, R. D. Piner, K. A. Kohlhaas, A. Kleinhammes, Y. Jia, Y. Wu, S. T. Nguyen and R. S. Ruoff, Carbon, 2007, 45, 1558-1565.

5 P.-G. Ren, D.-X. Yan, X. Ji, T. Chen and Z.-M. Li, Nanotechnology, 2010, 22, 055705.

6 H. J. Shin, K. K. Kim, A. Benayad, S. M. Yoon, H. K. Park, I. S. Jung, M. H. Jin, H. K. Jeong, J. M. Kim and J. Y. Choi, Adv. Funct. Mater., 2009, 19, 1987-1992.

7 Y. Wang, P. Zhang, C. F. Liu, L. Zhan, Y. F. Li and C. Z. Huang, RSC Adv., 2012, 2, 2322-2328.

8 D. Mhamane, W. Ramadan, M. Fawzy, A. Rana, M. Dubey, C. Rode, B. Lefez, B. Hannoyer and S. Ogale, Green Chem., 2011, 13, 1990-1996.

9 M. Nasrollahzadeh, M. Maham, A. Rostami-Vartooni, M. Bagherzadeh and S. M. Sajadi, RSC Adv., 2015, 5, 64769-64780.

10 E. C. Salas, Z. Sun, A. Lüttge and J. M. Tour, ACS Nano, 2010, 4, 4852-4856.

11 S. Roy and T. K. Das, Int J Plant Biol Res, 2015, 3, 1044.

12 A. Singh, D. Jain, M. Upadhyay, N. Khandelwal and H. Verma, Dig J Nanomater Bios, 2010, 5, 483-489.

13 C. Namasivayam, N. Muniasamy, K. Gayatri, M. Rani and K. Ranganathan, Bioresour. Technol., 1996, 57, 37-43.
14 S. Thakur and N. Karak, Carbon, 2012, 50, 5331-5339.

15 R. K. Upadhyay, N. Soin, G. Bhattacharya, S. Saha, A. Barman and S. S. Roy, Mater. Lett., 2015, 160, 355-358.

16 A. H. Al-Marri, M. Khan, M. Khan, S. F. Adil, A. AlWarthan, H. Z. Alkhathlan, W. Tremel, J. P. Labis, M. R. H. Siddiqui and M. N. Tahir, Int. J. Mol. Sci., 2015, 16, 1131-1142.

17 F. Lorestani, Z. Shahnavaz, P. Mn, Y. Alias and N. S. Manan, Sens. Actuators, B, 2015, 208, 389-398.

18 M. Vinothkannan, C. Karthikeyan, A. R. Kim and D. J. Yoo, Spectrochim. Acta, Part A, 2015, 136, 256-264.

19 X. Qin, W. Lu, A. M. Asiri, A. O. Al-Youbi and X. Sun, Catal. Sci. Technol., 2013, 3, 1027-1035.

20 K. J. Babu, K. S. Nahm and Y. J. Hwang, RSC Adv., 2014, 4, 7944-7951.

21 Z. Bo, X. Shuai, S. Mao, H. Yang, J. Qian, J. Chen, J. Yan and K. Cen, Sci. Rep., 2014, 4, 4684.

22 Y. Zhang, J. Tian, H. Li, L. Wang, X. Qin, A. M. Asiri, A. O. AlYoubi and X. Sun, Langmuir, 2012, 28, 12893-12900.

23 J.-L. Gong, B. Wang, G.-M. Zeng, C.-P. Yang, C.-G. Niu, Q.-Y. Niu, W.-J. Zhou and Y. Liang, J. Hazard. Mater., 2009, 164, 1517-1522.

24 M. Yusuf, F. Elfghi, S. A. Zaidi, E. Abdullah and M. A. Khan, RSC Adv., 2015, 5, 50392-50420.

25 P. Sharma, N. Hussain, D. J. Borah and M. R. Das, J. Chem. Eng. Data, 2013, 58, 3477-3488.

26 J. N. Tiwari, K. Mahesh, N. H. Le, K. C. Kemp, R. Timilsina, R. N. Tiwari and K. S. Kim, Carbon, 2013, 56, 173-182.

27 D. Suresh, H. Nagabhushana and S. Sharma, Mater. Lett., 2015, 142, 4-6.

28 D. Suresh, M. P. Kumar, H. Nagabhushana and S. Sharma, Mater. Lett., 2015, 151, 93-95.

29 R. Rajeswari, M. Umadevi, C. S. Rahale, S. Selvavenkadesh, K. S. Kumar and D. Bhowmik, J. Pharmacogn. Phytochem., 2012, 1, 118-124.

30 J. H. Hamman, Molecules, 2008, 13, 1599-1616.

31 S. P. Chandran, M. Chaudhary, R. Pasricha, A. Ahmad and M. Sastry, Biotechnology progress, 2006, 22, 577-583.

32 S. Maensiri, P. Laokul, J. Klinkaewnarong, S. Phokha, V. Promarak and S. Seraphin, J. Optoelectron. Adv. Mater., 2008, 10, 161-165.

33 M. Khan, A. H. Al-Marri, M. Khan, M. R. Shaik, N. Mohri, S. F. Adil, M. Kuniyil, H. Z. Alkhathlan, A. Al-Warthan and W. Tremel, Nanoscale Res. Lett., 2015, 10, 281.

34 M. Aunkor, I. Mahbubul, R. Saidur and H. Metselaar, RSC $A d v .$, 2016, 6, 27807-27828.

35 T. Chen, B. Zeng, J. Liu, J. Dong, X. Liu, Z. Wu, X. Yang and Z. Li, J. Phys.: Conf. Ser., 2009, 188, 1.

36 D. R. Dreyer, S. Park, C. W. Bielawski and R. S. Ruoff, Chem. Soc. Rev., 2010, 39, 228-240.

37 S. Song, Y. Ma, H. Shen, M. Zhang and Z. Zhang, RSC Adv., 2015, 5, 27922-27932.

38 H. Genç, J. C. Tjell, D. McConchie and O. Schuiling, J. Colloid Interface Sci., 2003, 264, 327-334.

39 V. Loryuenyong, K. Totepvimarn, P. Eimburanapravat, W. Boonchompoo and A. Buasri, Adv. Mater. Sci. Eng., 2013, 2013, 923403. 
40 F. Yakuphanoglu, S. Ilican, M. Caglar and Y. Caglar, J. Optoelectron. Adv. Mater., 2007, 9, 2180.

41 G. Wang, J. Yang, J. Park, X. Gou, B. Wang, H. Liu and J. Yao, J. Phys. Chem. C, 2008, 112, 8192-8195.

42 S. Srivastava, V. Kumar, M. A. Ali, P. R. Solanki, A. Srivastava, G. Sumana, P. S. Saxena, A. G. Joshi and B. Malhotra, Nanoscale, 2013, 5, 3043-3051.

43 G. Bhattacharya, G. Kandasamy, N. Soin, R. K. Upadhyay, S. Deshmukh, D. Maity, J. McLaughlin and S. S. Roy, RSC Adv., 2017, 7, 327-335.

44 G. Bhattacharya, S. Pal, J. McLaughlin and S. S. Roy, Int. J. Electrochem. Sci., 2016, 11, 6370-6386.

45 V. Varade, G. V. Honnavar, P. Anjaneyulu, K. Ramesh and R. Menon, J. Phys. D: Appl. Phys., 2013, 46, 365306.

46 G. Ramesha, A. V. Kumara, H. Muralidhara and S. Sampath, J. Colloid Interface Sci., 2011, 361, 270-277.

47 Z. Geng, Y. Lin, X. Yu, Q. Shen, L. Ma, Z. Li, N. Pan and X. Wang, J. Mater. Chem., 2012, 22, 3527-3535.

48 H. Yuh-Shan, Scientometrics, 2004, 59, 171-177.

49 Y.-S. Ho and G. McKay, Chem. Eng. J., 1998, 70, 115-124.
50 W. J. Weber and J. C. Morris, J. Environ. Eng. Div., 1963, 89, 31-60.

51 W. Zou, K. Li, H. Bai, X. Shi and R. Han, J. Chem. Eng. Data, 2011, 56, 1882-1891.

52 L. Ai, M. Li and L. Li, J. Chem. Eng. Data, 2011, 56, 3475-3483.

53 H. Sun, L. Cao and L. Lu, Nano Res., 2011, 4, 550-562.

54 W. Fan, W. Gao, C. Zhang, W. W. Tjiu, J. Pan and T. Liu, J. Mater. Chem., 2012, 22, 25108-25115.

55 P. K. Sahu, D. D. Giri, R. Singh, P. Pandey, S. Gupta, A. K. Shrivastava, A. Kumar and K. D. Pandey, Pharmacol. Pharm., 2013, 4, 599.

56 H. Sun, S. Liu, G. Zhou, H. M. Ang, M. O. Tadé and S. Wang, ACS Appl. Mater. Interfaces, 2012, 4, 5466-5471.

57 Z. Xiong, L. L. Zhang and X. S. Zhao, Chem.-Eur. J., 2011, 17, 2428-2434.

58 N. Van Hoa, T. T. Khong, T. T. H. Quyen and T. S. Trung, Journal of Water Process Engineering, 2016, 9, 170-178.

59 D. Dutta, S. Thiyagarajan and D. Bahadur, Chem. Eng. J., 2016, 297, 55-65. 